\title{
Insulating Phases and Superfluid-Insulator Transition of Disordered Boson Chains
}

\author{
Ehud Altman, ${ }^{1}$ Yariv Kafri, ${ }^{2}$ Anatoli Polkovnikov, ${ }^{3}$ and Gil Refael ${ }^{4}$ \\ ${ }^{1}$ Department of Condensed Matter Physics, The Weizmann Institute of Science Rehovot, 76100 Israel \\ ${ }^{2}$ Department of Physics, Technion, Haifa 32000, Israel \\ ${ }^{3}$ Department of Physics, Boston University, Boston, Massachusetts 02215, USA \\ ${ }^{4}$ Department of Physics, California Institute of Technology, MC 114-36, Pasadena, California 91125, USA \\ (Received 23 November 2007; revised manuscript received 28 January 2008; published 1 May 2008)
}

\begin{abstract}
Using a strong disorder real-space renormalization group, we study the phase diagram of a fully disordered chain of interacting bosons. Since this approach does not suffer from runaway flows, it allows a direct study of the insulating phases, not accessible in a weak disorder perturbative treatment. We find that the universal properties of the insulating phase are determined by the details and symmetries of the on-site chemical-potential disorder. Three insulating phases are possible: (i) an incompressible Mott glass with a finite superfluid susceptibility, (ii) a random-singlet glass with diverging compressibility and superfluid susceptibility, (iii) a Bose glass with a finite compressibility but diverging superfluid susceptibility. In addition to characterizing the insulating phases, we show that the superfluid-insulator transition is always described by Kosterlitz-Thouless-like flows.
\end{abstract}

DOI: 10.1103/PhysRevLett.100.170402

PACS numbers: 05.30.Jp, 74.81.- $\mathrm{g}$

Bose systems can be driven into an insulating phase by quantum fluctuations due to strong repulsive interactions and lattice effects. The impact of a disordered potential on this superfluid-insulator transition and on the nature of the insulating phases is a long standing question [1]. In weakly disordered chains, the momentum-shell renormalization group (RG) afforded much progress [2,3]. Recently, however, an analysis using a real-space RG suggested that strong disorder can have very different effects on a one dimensional Bose system [4]. In particular, it was found that a certain disorder type, which is perturbatively irrelevant, can induce a transition when sufficiently strong, and lead to a new kind of an insulator termed the Mott glass $[4,5]$. The existence of this Mott glass was confirmed numerically [6,7]. The disorder considered in Refs. [4,6], however, had a very special particle-hole symmetry properties not easily realizable in actual experiments (e.g., $[8,9])$.

In this Letter we extend the real-space RG of Ref. [4] to treat strong and general disorder potentials, not confined to the comensurability requirement in Ref. [4]. Our starting point is the disordered quantum-rotor model:

$$
\mathcal{H}=\sum_{j} \frac{U_{j}}{2}\left(\hat{n}_{j}-\bar{n}_{j}\right)^{2}-\sum_{j} J_{j} \cos \left(\varphi_{j+1}-\varphi_{j}\right),
$$

which describes a chain of superfluid grains, connected by a random Josephson coupling $J_{j}$. Each grain has a random charging energy $U_{j}$, and offset-charge $\bar{n}_{j}$, representing an excess screening charge on the site or in its environment, and parameterizes a random on-site chemical potential $\mu_{j}=U_{J} \bar{n}_{j}$. The lattice model (1) gives also a coarsegrained description of continuum bosons, with grain size set by the healing length of the condensate.

Using real-space RG, we obtain the phase diagram of the fully disordered bosnic chain, and show that it can undergo a transition from a superfluid to three possible insulating phases, whose nature depends on the symmetry properties of the offset-charge distribution. Unlike momentum-shell RG [2], our treatment does not have runaway flows, and allows quantitative analysis of the insulating phases. We characterize the insulators using the charging gap $\Delta$, the compressibility $\kappa=\frac{\partial n}{\partial \mu}$ and the superfluid susceptibility $\chi_{s}$ (i.e., the linear response of the order parameter $\overline{\left\langle e^{i \varphi_{j}}\right\rangle}$ to the coupling $\frac{\delta}{L} \sum_{i} \cos \varphi_{i}$, with angular brackets and overline denoting, respectively, quantum expectation value and disorder average). The three insulators are illustrated schematically in Fig. 1. They include: (i) an incompressible Mott glass arising for the case of zero offset charges $\bar{n}_{j}=$ 0 ; (ii) a glass phase with a diverging compressibility which arises if $\bar{n}_{j}$ is restricted to 0 or $1 / 2$, and which we term a random-singlet glass; and (iii) a Bose-glass phase characterized by a finite compressibility and a diverging superfluid susceptibility in the case of a generic, nonsingular, offset-charge distribution in the range $-1 / 2<\bar{n}_{j} \leq 1 / 2$. While the nature of the insulators depends strongly on the symmetry properties of the offset-charge disorder, we show that the superfluid phase and the phase transition at strong disorder are insensitive to these details.

Our real-space RG is carried out by eliminating the highest energy scale in the system at each stage via a local decimation step [10-12]. The Hamiltonian keeps its form [Eq. (1)], but with renormalized distributions of $J_{i}, U_{i}$ and $\bar{n}_{i}$, which turn out to be universal, i.e., independent of the details of the bare disorder distributions. The universal distributions and their flow yield a wealth of information, especially near the little understood superfluid-insulator transition at strong disorder. From them we derive the system's scaling and thermodynamic properties.

Generalized decimation procedure. - Let us define the global energy scale $\Omega=\max _{j}\left(\Delta_{j}, J_{j}\right)$, where 


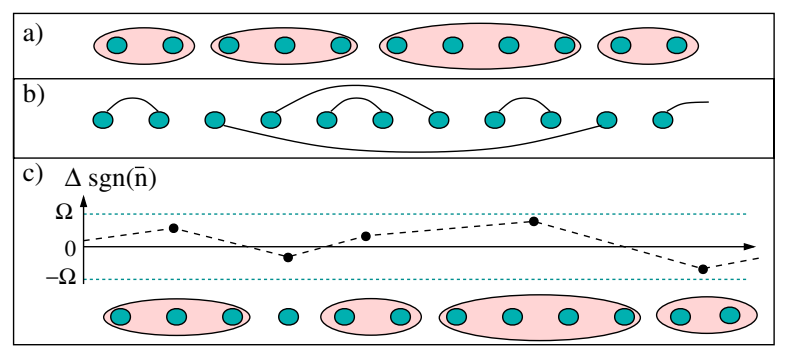

FIG. 1 (color online). The three insulating phases that emerge for different classes of disorder. (a) The Mott glass is realized when only the Josephson couplings and charging energies are disordered, and with no offset charge, $\bar{n}=0$. At large scales it consists of effectively disconnected superfluid clusters of random size. (b) The "random-singlet" glass appears when the random offset charge is restricted to $\bar{n}=0,1 / 2$. In this phase bosons are delocalized on random pairs of remote clusters. (c) The Bose glass is realized for a generic offset-charge distribution. In it, large superfluid clusters act effectively as weakly coupled spin-1/2's in a uniformly-distributed random $z$ field, given by the on-site gap times $\bar{n}$ 's sign: $\Delta \operatorname{sgn}(\bar{n})=U(1-$ $2|\bar{n}|) \operatorname{sgn}(\bar{n})$.

$\Delta_{j}=U_{j}\left(1-2\left|\bar{n}_{j}\right|\right)$ is the charging energy of the site $j$. For the Hamiltonian in Eq. (1) three types of decimation steps are possible. Type 1: site decimation. If $\Omega=\Delta_{j}$ for some $j$, we freeze the charge on the site $j$, thus eliminating this degree of freedom. A Josephson coupling $J_{j-1} \approx$ $J_{j} J_{j+1} / \Omega$ between the sites $j-1$ and $j+1$ is generated by a virtual tunneling process through the eliminated site (see Ref. [4]). Type 2: Bond decimation. If $\Omega=J_{j}$ for some $j$, sites $j$ and $j+1$ merge into a superfluid cluster with an effective interaction parameter: $1 / \tilde{U}_{j}=1 / U_{j}+$ $1 / U_{j+1}$ (i.e., parallel capacitances add up). The offset charges of the two sites simply add up $\tilde{\bar{n}}_{j}=\bar{n}_{j}+\bar{n}_{j+1}$. Type 3: Doublet formation. A special RG step is introduced for sites with the offset-charge $\bar{n}_{j}=1 / 2$. Here, if $U_{j}>\Omega$ the site $j$ is frozen to its two lowest-lying degenerate charge states. Then we can set $U_{j} \rightarrow \infty$ and treat the site as a spin- $1 / 2$ degree of freedom (spin-site) with $s_{j}^{z}=$ $\left(n_{j}-\bar{n}_{j}\right)$ and, similarly, $\exp \left( \pm i \phi_{j}\right) \rightarrow \hat{s}_{j}^{ \pm}$. The spin sites require revisiting the bond decimation step (Type 2). If the strong bond connects a spin site to a regular site, then the spin site can be simply treated as a half-integer site with infinite $U$. But when two spin sites are strongly coupled by large $J$ corresponding to $x y$ coupling: $-J_{j}\left(s_{j}^{x} s_{j+1}^{x}+\right.$ $\left.s_{j}^{y} s_{j+1}^{y}\right)$, they freeze into the triplet state with $m_{z}=0: \frac{1}{\sqrt{2}} \times$ $\left(\left|\uparrow_{j} \downarrow_{j+1}\right\rangle+\left|\uparrow_{j} \downarrow_{j+1}\right\rangle\right)$. This represents a boson resonating in a symmetric superposition between the two sites, with a "charging" gap $J_{j}$ to the excited (antisymmetric) state. Quantum fluctuations now allow tunneling between sites $j-1$ and $j+2$ with strength $J_{j-1} J_{j+1} / J_{j}$. The last step is identical to the real-space RG in the random $x x$ spin chain [12].

The RG flow can lead to either a superfluid or an insulating phase. In the superfluid, the system coalesces to a large superfluid cluster, while in the insulator, it breaks down to clusters with large effective charging gaps connected by weak tunneling. Quantitative analysis of the RG requires the solution of integro-differential equations for the disorder distributions [12]. Remarkably, for the disorder type we consider, the distributions of $U_{i}, J_{i}$, and $\bar{n}_{i}$, are universal in a large vicinity around the RG's fixed points, which govern the superfluid-insulator transitions. This greatly simplifies the flow equations, and in several cases allows an analytical solution. Below we discuss the three classes of disorder, corresponding to different symmetry properties of the offset-charge distribution.

No offset charge. -The case of $\bar{n}_{j}=0$, where the Hamiltonian (1) is particle-hole symmetric, was analyzed in Ref. [4]. For completeness, we review the main results. The RG steps (here only the decimations of types 1 and 2 are needed) translate into the flow of the coupling distributions $F(U / \Omega)$ and $G(J / \Omega)$. Near the superfluidinsulator fixed point they acquire the universal form:

$$
F(x) \approx \frac{A}{x^{2}} \exp \left[-\frac{f_{0}(\Omega)}{x}\right], \quad G(x) \approx g_{0}(\Omega) x^{g_{0}(\Omega)-1} .
$$

Here $A$ is a normalization constant, and $x \leq 1$ [13]. The typical Josephson coupling and site charging energies are monotonic functions of $g_{0}$ and $f_{0}$ respectively. In [4] we derived the flow of $g_{0}$ and $f_{0}$ :

$$
\frac{d f_{0}}{d \Gamma}=f_{0}\left(1-g_{0}\right), \quad \frac{d g_{0}}{d \Gamma}=-f_{0} g_{0},
$$

where $\Gamma=\ln \left(\Omega_{0} / \Omega\right)$ and $\Omega_{0}$ is the initial cutoff energy scale. The solutions of (3) are parametrized by the constant $C: f_{0} \approx C+\left(1-g_{0}\right)^{2} / 2$. $C<0$ corresponds to the superfluid state where the charging energy is irrelevant $\left(f_{0}\right.$ flows to zero) and $g_{0}$ flows to constant $g_{0}>1$. $C>0$ describes the insulator: $f_{0}$ is relevant $\left(f_{0} \rightarrow \infty\right)$ and $g_{0}$, indicative of the typical Josephson coupling strength, flows to zero. $C=$ 0 marks the critical point separating the two phases. At this point $g_{0}$ flows to 1 and $f_{0}$ flows to zero. We note that substituting $f_{0} \rightarrow y_{0}^{2}$ makes Eqs. (3) assume a standard Kosterlitz-Thouless form.

The fixed point in the superfluid phase corresponds to a classical model with $U_{i} \equiv 0$. The fixed point being noninteracting, implies neither the vanishing of the compressibility, nor the formation of true long range order: since our analysis relies on the grand canonical ensemble, the lowest excitation in the superfluid is an addition of a particle and not a phase twist or a Bogoliubov excitation. Thus the vanishing of $U_{j}$ only implies that the energy for adding a particle vanishes with the inverse system size, as expected in a superfluid. Obtaining the compressibility or stiffness of the superfluid requires a more detailed analysis, that keeps track of the internal Josephson couplings and phonon modes within renormalized clusters.

In this Letter we concentrate on the properties of the insulating phases, which are most drastically affected by 
the type of the disorder. In the insulating phases, the canonical and grand canonical pictures of the excitations are identical, and they are described correctly by the RG. The insulating phases are best described by a chain consisting of nearly disconnected clusters, each with its own charging gap. The lowest gap corresponds to the energy scale $\Omega$ at which the last site is decimated. From Ref. [4], in the commensurate case $\left(\bar{n}_{j}=0\right)$ this gap vanishes with system size $L$ as $\Delta \sim \frac{1}{\ln L}$ and is governed by rare and anomalously large superfluid clusters. The compressibility vanishes as $(\ln L) / L$, and the superfluid susceptibility is finite. We termed this gapless incompressible phase a Mottglass. This phase was also discussed in Refs. [5,14], and confirmed numerically in Refs. [6,7].

Mixed offset. $-\overline{\mathbf{n}}=(\mathbf{0}, \mathbf{1} / \mathbf{2})$. Let us now allow charge offsets, but still maintain the particle-hole symmetry of the Hamiltonian (1), i.e., its invariance under the transformation $n_{j} \rightarrow 1-n_{j}$. Such a restriction naturally arises in small superconducting grains with a pairing gap much larger than charging energy. The random parity of the electron number leads to an offset charge which is randomly integer or half-integer the cooper-pair charge.

In addition to distributions of $U$ and $J$, we must now also follow $\bar{n}$ 's distribution. We parametrize it by the three probabilities - $q, p$, and $s-$ corresponding to relative densities of integer $\left(\bar{n}_{j}=0\right)$, half-integer $\left(\bar{n}_{j}=1 / 2\right)$, and doublet (spin-1/2) sites, respectively. The flow of the $\bar{n}$ distribution is given through flow equations for $p$ and $q$ (note that $p+q+s=1$ due to normalization). Postponing a detailed description of these equations, let us here observe that whether an effective cluster has $\bar{n}_{j}=0$ or $1 / 2$, depends only on the parity of the number of the bare sites with $\bar{n}=1 / 2$ contained in it. This implies that no matter what the fraction of the half-integer sites in the original chain was (as long as it is nonzero) large clusters have odd or even parity with equal probabilities. For this reason the distribution quickly flows to a fixed line with $p=q=(1-s) / 2$, provided $p>0$ initially.

This observation significantly simplifies the flow analysis, and reduces the number of flow equations to three. From the integrodifferential equations we find that the universal distributions of $J$ and $U$ given by Eqs. (2) still hold, and we obtain a simplified set of flow equations:

$$
\begin{aligned}
\frac{d f_{0}}{d \Gamma} & =f_{0}\left[1-g_{0}(1-s)\left(1+f_{0}\right)\right], \\
\frac{d g_{0}}{d \Gamma} & =-\frac{g_{0}}{2}\left[(1-s) f_{0}+2 s^{2} g_{0}\right], \\
\frac{d s}{d \Gamma} & =\frac{f_{0}}{2}\left(1-s^{2}\right)-g_{0} s(1-s) .
\end{aligned}
$$

These have two different families of solutions, each marking a phase. In one family, the flow is to a stable fixed line with $s=0, f_{0}=0$ and $g_{0}>1$ : a superfluid phase identical to that of the $\bar{n}_{j}=0$ case. Close to this line, the flow equations reduce to Eqs. (3), except for an extra 1/2 in the equation for $g_{0}$, which appears since only sites with $\bar{n}=0$ renormalize $J$. The unimportance of the random offset in the superfluid is not surprising: local density fluctuations screen the offset charge.

The superfluid-insulator transition is also very similar to the $\bar{n}=0$ case, and it is described by essentially the same Kosterlitz-Thouless-like critical point with $g_{0}=1, f_{0}=0$ and $s=0$, similar to Eqs. (3). In the insulating phase at $g_{0}<1$, however, $f_{0}$ and the spin-site density $s$ become relevant, and $s$ quickly flows to 1 . This second family of solutions of (4) describes a different insulating phase than in the case of $\bar{n}_{j}=0$; it corresponds to an effective spin- $1 / 2$ chain with random $x$ - $y$ ferromagnetic couplings, a model analyzed in detail in Ref. [12]. Its ground state consists of random noncrossing pairs of sites at varying distances, in which the spins form the $m_{z}=0$ state, $\frac{1}{\sqrt{2}} \times$ $(|\uparrow \downarrow\rangle+|\downarrow \uparrow\rangle)$. This phase is termed the random-singlet glass, following the random-spin chain term $[15,16]$. In the bosonic language the ground state has bosons delocalized randomly between pairs of sites.

Many properties of the random-singlet glass can be inferred directly from Ref. [12]. The energy scale associated with breaking a singlet between sites of distance $\ell$ is $\Omega_{\ell}=\Omega_{0} \exp (-\sqrt{\ell})$. By setting $\ell=L$, the system size, we get the scaling of the gap vs $L$. Following the identification $n_{i}=\frac{1}{2}+\hat{s}_{i}^{z}$, the compressibility $\kappa$ and superfluid susceptibility $\chi_{s}$ in the insulating phase correspond to the susceptibilities of a random-spin chain to a Zeeman field in the $z$ and $x$ directions, respectively. Reference [12] shows that both susceptibilities diverge at the limit of small $\Omega$ as $\kappa(\Omega) \sim \chi_{s}(\Omega) \sim 1 / \Omega \log ^{3}\left(\Omega_{0} / \Omega\right)$. While the superfluid stiffness vanishes in the thermodynamic limit, unlike the stiffness of the Mott glass, it vanishes only subexponentially with $L: \rho_{s} \propto e^{-\sqrt{L}}$. Also note that $g_{0}$, indicative of the Josephson coupling strength, flows to zero as $g_{0} \sim 1 / \Gamma$ [cf. Eq. (4) with $s=1$ ], much slower than $g_{0} \sim$ $\exp \left[e^{-\Gamma}\right]=\exp [-1 / \Omega]$ in the Mott glass.

Generic chemical potential disorder.-When all offsets $-1 / 2<\bar{n}_{j} \leq 1 / 2$ are allowed, the relevant energy scale for the RG site decimation is not $U$, but rather the local gap $\Delta_{i}=U_{i}\left(1-2\left|\bar{n}_{i}\right|\right)$. The interaction $U_{i}$ is allowed to exceed $\Omega$ so long that $\Delta_{i}<\Omega$ :

$$
U_{j}<\Omega /\left(1-2\left|\bar{n}_{j}\right|\right) .
$$

Thus we must consider a joint distribution for $U$ and $\bar{n}$. Despite this complication, our analysis reveals a rather intuitive behavior, which we derive succinctly below.

First, note that $\bar{n}$ disorder width is a relevant variable due to the rule $\bar{n}_{j} \rightarrow \bar{n}_{j}+\bar{n}_{j+1}$ for a bond decimation. Therefore as the effective sites grow with the RG, their offset charges quickly become uniformly distributed between $-1 / 2$ and $1 / 2$, i.e., the largest disorder allowed. This observation simplifies the analysis significantly. In particular, it is straightforward to check that the distributions of $U$ and $J$ again approach the universal functions (2). In the superfluid regime and at the transition point the flow is also governed by the Eqs. (3) with an extra $1 / 2$ in the $g_{0}$ 
equation. Thus the system with generic disorder undergoes the same transition as in the two cases discussed above. Again, at weak interactions, the offset-charge disorder is screened by large particle-number fluctuations. In the insulating side, $f_{0}$ flows to large values; the joint distribution of $U / \Omega$ and $\bar{n}$ is still given by (2), $F^{\text {joint }}(U / \Omega, \bar{n})=$ $F(U / \Omega)$, but with the upper bound of $U / \Omega$ obeying Eq. (5). At large $f_{0}$, this form of $F^{\text {joint }}(U / \Omega, \bar{n})$ leads to the uniform distribution of the charging gaps $\Delta$ of each site: $H(\Delta) \approx 1 / \Omega$. Most importantly, $H(\Delta)$ is nonsingular at $\Delta \rightarrow 0$.

Deep in the insulating phase, since the typical charging energy $U$ is large, each site is akin to a doublet of states which is split locally by the energy $\Delta<\Omega$, while all other states lie at energies above the cutoff. When $\Delta=0$ this doublet is degenerate and represents the spin- $1 / 2$ degree of freedom discussed above. Thus the spin- $1 / 2$ description applies also to the case of nonzero $\Delta$ with the latter playing the role of the Zeeman field along the $z$ axis. The effective Hamiltonian for the chain becomes

$$
\mathcal{H}=-\sum_{j} \tilde{J}_{j}\left(s_{j}^{x} s_{j+1}^{x}+s_{j}^{y} s_{j+1}^{y}\right)-\sum_{j} \Delta_{j} s_{j}^{z},
$$

where $s_{i}^{z}=n_{i}-1 / 2$, and here $\Delta_{j}=U_{j}\left(1-2\left|\bar{n}_{j}\right|\right) \operatorname{sgn}\left(\bar{n}_{j}\right)$ can have either sign, and the distribution $H[\Delta \operatorname{sgn}(\bar{n})]=$ $1 / 2 \Omega$. Because $\tilde{J}_{j} \ll \Omega$ we can calculate many properties of this phase by considering a single site in a random Zeeman field. First, the gap distribution implies that in this phase the energy-length scaling is $\Omega_{L} \sim 1 / L=\rho$. Next, the compressibility is given by the response to an external $z$ field: $\kappa=\rho \partial \overline{\left\langle s^{z}\right\rangle} / \partial h_{\mathrm{ext}}^{z}=2 H(0)=\rho / \Omega=\kappa_{0}$, a constant at low energies. The superfluid susceptibility is the response to a transverse field: $\chi_{s}=\rho \partial \overline{\left\langle s^{x}\right\rangle} / \partial h_{\mathrm{ext}}^{x}$. We find this disorder average from the distribution of $\Delta_{i}$ :

$$
\chi_{s}=\rho \int_{-\Omega}^{\Omega} \frac{d \Delta}{2 \Omega} \frac{1}{\sqrt{\left(h_{\mathrm{ext}}^{x}\right)^{2}+\Delta^{2}}} \approx \frac{\kappa_{0}}{2} \log \left(\frac{\Omega}{\left|h_{\mathrm{ext}}^{x}\right|}\right),
$$

which diverges as $h_{\mathrm{ext}}^{x} \rightarrow 0$, as dictated by $H(\Delta)$ being nonsingular. In a finite chain this divergence is cut off by the smallest $\left|\Delta_{j}\right|$, and leads to $\chi_{s} \sim \log L$. The finite compressibility and diverging superfluid susceptibility coincide with those of the Bose glass phase discussed in Ref. [1], and numerically observed in Ref. [17].

In summary, we extended the real-space RG analysis of Ref. [4] to address noncommensurate random chemical potential. We found that the symmetry of the Hamiltonian and the details of the disorder, as encoded in the distribution of the offset $\bar{n}_{j}$, do not affect the universal properties of the superfluid phase and superfluid-insulator transition, but at the same time it completely determines the insulating phase of the system. Alongside the Mott glass of the $\bar{n}_{j}=0$ case, we find the random-singlet glass for the mixed $\bar{n}=0,1 / 2$ case, and for generic chemicalpotential disorder, a phase identified as the Bose glass [1]. All our results are supported by a numerical real-space RG study to be published separately.
TABLE I. Gap $\Delta$, compressiblity $\kappa$, and SF susceptibility $\chi_{s}$, of the insulators realized for the different classes of disorder in offset $\bar{n}$. $L$ is the system size, and $c$ is a nonuniversal constant.

\begin{tabular}{lcccc}
\hline \hline $\bar{n}$ disorder & Glass type & $\Delta$ & $\kappa$ & $\chi_{s}$ \\
\hline 0 & Mott & $\frac{1}{\log L}$ & $\frac{\log L}{L} \rightarrow 0$ & const \\
$0,1 / 2$ & Rand-singlet & $e^{-c \sqrt{L}}$ & $\frac{1}{L^{3 / 2}} e^{c \sqrt{L}}$ & $\frac{1}{L^{3 / 2}} e^{c \sqrt{L}}$ \\
$-1 / 2 \leq \bar{n}<1 / 2$ & Bose & $\frac{1}{L}$ & $\kappa$ & $\frac{\kappa}{2} \log L$ \\
\hline \hline
\end{tabular}

Our focus was the universal thermodynamic properties of the insulating phases, summarized in Table I. An outstanding question, left for future publication, is the effective Luttinger parameter at the critical point, and how it compares to the low-disorder motivated results of Ref. [2], predicting $K=\sqrt{\kappa \rho_{s}}=3 / 2$. Our initial results, however, indicate that for sufficiently strong disorder the transition we discuss differs from its weak disorder analog, and occurs at a nonuniversal $K$, which depends on disorder strength and exceeds $3 / 2$. This possibility does not contradict the thermodynamic argument of Ref. [6].

We thank S. Girvin for suggesting considering the halfinteger case. We also acknowledge T. Giamarchi, P. LeDoussal, O. Motrunich, N. Prokofe'v, and B. Svistunov for numerous discussions. A. P.'s research was supported by AFOSR YIP, Y.K. and G.R. acknowledge Boston University visitors program's hospitality. Y. K. and E. A. thank the ISF and BSF for support.

[1] M. P. A. Fisher, P. B. Weichman, G. Grinstein, and D. S. Fisher, Phys. Rev. B 40, 546 (1989).

[2] T. Giamarchi and H. J. Schulz, Europhys. Lett. 3, 1287 (1987).

[3] T. Giamarchi and H.J. Schulz, Phys. Rev. B 37, 325 (1988).

[4] E. Altman, Y. Kafri, A. Polkovnikov, and G. Refael, Phys. Rev. Lett. 93, 150402 (2004).

[5] T. Giamarchi, P. Le Doussal, and E. Orignac, Phys. Rev. B 64, 245119 (2001).

[6] K. G. Balabanyan, N. Prokof'ev, and B. Svistunov, Phys. Rev. Lett. 95, 055701 (2005).

[7] P. Sengupta and S. Haas, Phys. Rev. Lett. 99, 050403 (2007).

[8] B. Demarco (private communication).

[9] J.E. Lye et al., Phys. Rev. Lett. 95, 070401 (2005).

[10] S. K. Ma, C. Dasgupta, and C. K. Hu, Phys. Rev. Lett. 43, 1434 (1979).

[11] C. Dasgupta and S. K. Ma, Phys. Rev. B 22, 1305 (1980).

[12] D. S. Fisher, Phys. Rev. B 50, 3799 (1994).

[13] For the commensurate case $A=e^{f_{0}} / f_{0}$, but for generic disorder, where $0<x<\infty, A$ has a different value.

[14] E. Orignac, T. Giamarchi, and P. Le Doussal, Phys. Rev. Lett. 83, 2378 (1999).

[15] R. N. Bhatt and P. A. Lee, Phys. Rev. Lett. 48, 344 (1982).

[16] C. A. Doty and D. S. Fisher, Phys. Rev. B 45, 2167 (1992).

[17] R. T. Scalettar, G. G. Batrouni, and G. T. Zimanyi, Phys. Rev. Lett. 66, 3144 (1991). 